\title{
A Generalized Piezoelectric Bernoulli-Navier Anisotropic Rod Model
}

\author{
Isabel M. Narra Figueiredo • Carlos M. Franco Leal
}

Received: 23 December 2005 / Accepted: 26 May 2006 /

Published online: 25 July 2006

(C) Springer Science+Business Media B.V. 2006

\begin{abstract}
We apply the asymptotic analysis procedure to the three-dimensional static equations of piezoelectricity, for a linear nonhomogeneous anisotropic thin rod. We prove the weak convergence of the rod mechanical displacement vectors and the rod electric potentials, when the diameter of the rod cross-section tends to zero. This weak limit is the solution of a new piezoelectric anisotropic nonhomogeneous rod model, which is a system of coupled equations, with generalized Bernoulli-Navier equilibrium equations and reduced Maxwell-Gauss equations.
\end{abstract}

Mathematics Subject Classifications (2000) $74 \mathrm{~K} 10 \cdot 74 \mathrm{G} 10 \cdot 78 \mathrm{~A} 30$

Key words asymptotic analysis $\cdot$ anisotropic material $\cdot$ piezoelectricity $\cdot \operatorname{rod}$

\section{Introduction}

In a previous paper we have mathematically justified a new nonhomogeneous anisotropic piezoelectric plate model (cf. Figueiredo and Leal [7]). This was achieved considering the three-dimensional static equations of piezoelectricity, and using the asymptotic analysis method to compute the limit of the plate mechanical displacement vectors and of the plate electric potentials, as the thickness of the plate approaches zero (see also Maugin and Attou [9], Rahmoune et al. [10], Sene [12],

I. M. Narra Figueiredo $(\varangle)$. C. M. Franco Leal

Departamento de Matemática, Universidade de Coimbra, Apartado 3008, 3001-454 Coimbra, Portugal

e-mail: isabelf@mat.uc.pt

URL: http//www.mat.uc.pt/ isabelf

C. M. Franco Leal

e-mail: carlosl@mat.uc.pt 
and Raoult and Sene [11] for works reporting different asymptotic models for piezoelectric plates, Collard and Miara [3] for asymptotic models for piezoelectric shells, and Ciarlet [2] for a detailed description of the asymptotic analysis procedure to elastic plates).

In the present paper, we consider again the three-dimensional static equations of piezoelectricity, but for a thin rod, whose cross-section is a function of a small parameter and made of a nonhomogeneous and anisotropic material. Our purpose is to use once more the asymptotic analysis to obtain the limit of the three-dimensional rod mechanical displacement vectors and rod electric potentials, as the diameter of the rod cross-section tends to zero (cf. also Trabucho and Viaño [13] for a description of the asymptotic analysis method for elastic rods). Thus the resulting asymptotic model constitutes a simplified rod model that may be used with confidence by engineers.

The technique used in this paper is very similar to that described in Figueiredo and Leal [7], and likewise the latter paper, one of the main difficulties is the anisotropy and the nonhomogeneity of the material: We suppose the material is monoclinic and nonhomogeneous and the piezoelectric and dielectric coefficients are functions and not constants.

The main result of the paper is the existence of a weak limit for the threedimensional rod mechanical displacement vectors and for the rod electric potentials, as the rod cross-section goes to zero (cf. Theorems 3.1, 3.2 and 3.3). In addition we show that this weak limit is the solution of a new nonhomogeneous anisotropic piezoelectric rod model (cf. Theorem 4.1, formulas (69)-(76), or equivalently, Theorem 4.2, formulas (103)-(110)), which can be briefly interpreted in the following way: It is a system of two coupled equations, with generalized Bernoulli-Navier equilibrium equations and reduced Maxwell-Gauss equations, where the limit rod mechanical displacement vector and the limit rod electric potential are interdependent. Unlike the plate case (cf. Figueiredo and Leal [7]) we were not able to determine the exact expression of the limit rod electric potential as a function of the limit rod mechanical displacement vector. In fact, in the limit rod problem there appears a third unknown that is coupled simultaneously with the limit mechanical displacement and the limit electric potential, which yields a very complex limit model. Moreover due to this complexity it was not possible to prove the uniqueness of solution for the limit rod problem.

To finish this introduction we briefly sketch the contents of the paper. In the next section, the three-dimensional piezoelectric rod model is introduced. The asymptotic analysis is done in Section 3; here a weak limit of the rod mechanical displacement vectors and the rod electric potentials is computed, and the variational formulation of the limit problem is described. Section 4 concerns the description of the differential formulation of the limit problem, firstly defined in the scaled rod and secondly in the original rod.

\section{The Three-Dimensional Piezoelectric Rod Model}

In this section we first introduce some notation. Then, we describe the boundary value problem associated with the static three-dimensional piezoelectric model, for a nonhomogeneous anisotropic rod, as well as, its corresponding variational formulation. 


\subsection{Notation}

Let $\omega \subset \mathbb{R}^{2}$ be a bounded domain with a Lipschitz continuous boundary $\partial \omega$ and $\gamma_{e}$ a subset of $\partial \omega$, such that, the measure of $\gamma_{e}$ satisfies meas $\left(\gamma_{e}\right)>0$.

For each real parameter $h$, with $0<h \leq 1$, we set

$$
\omega^{h}=h \omega, \quad \gamma^{h}=\partial \omega^{h}=h \partial \omega, \quad \gamma_{e}^{h}=h \gamma_{e},
$$

and

$$
\begin{aligned}
\Omega^{h} & =\omega^{h} \times(0, L), & & \Gamma_{0}^{h}=\omega^{h} \times\{0\}, \quad \Gamma_{L}^{h}=\omega^{h} \times\{L\}, \\
\Gamma_{D}^{h} & =\Gamma_{0}^{h} \bigcup \Gamma_{L}^{h}, & & \Gamma_{N}^{h}=\gamma^{h} \times(0, L), \\
\Gamma_{e D}^{h} & =\gamma_{e}^{h} \times(0, L), & \Gamma_{e N}^{h} & =\partial \Omega^{h} \backslash \Gamma_{e D}^{h} .
\end{aligned}
$$

The closure of the set $\Omega^{h}, \bar{\Omega}^{h}=\bar{\omega}^{h} \times[0, L]$, represents the rod, which has length $L$ and cross-section $\omega^{h}=h \omega$. The two extremities of the rod are $\Gamma_{0}^{h}$ and $\Gamma_{L}^{h}$, and the set $\Gamma_{N}^{h}$ is its lateral surface. The set $\Gamma_{e D}^{h}$ is a portion of the lateral surface of the rod and finally $\Gamma_{e N}^{h}$ is a part of the boundary $\partial \Omega^{h}$ of $\Omega^{h}$, which is disjoint from $\Gamma_{e D}^{h}$. An arbitrary point of $\Omega^{h}$ is denoted by $x^{h}=\left(x_{1}^{h}, x_{2}^{h}, x_{3}^{h}\right)$ where $\left(x_{1}^{h}, x_{2}^{h}\right)=\left(h x_{1}, h x_{2}\right)$, with $\left(x_{1}, x_{2}\right) \in \omega$, and the third component $x_{3}^{h}=x_{3} \in(0, L)$ is independent of $h$. We also denote by $v^{h}=\left(v_{1}^{h}, v_{2}^{h}, v_{3}^{h}\right)$ the outward unit normal vector to $\partial \Omega^{h}$. Moreover, we assume that the fixed coordinate system $O X_{1} X_{2} X_{3}$ is a principal system of inertia associated with $\omega \times(0, L)$;

$$
\int_{\omega} x_{1} d \omega=\int_{\omega} x_{2} d \omega=\int_{\omega} x_{1} x_{2} d \omega=0 .
$$

Throughout the paper, Latin indices $i, j, k, l \ldots$ belong to the set $\{1,2,3\}$, Greek indices $\alpha, \beta, \mu \ldots$ vary in the set $\{1,2\}$ and the summation convention with respect to repeated indices is employed that is $a_{i} b_{i}=\sum_{i=1}^{3} a_{i} b_{i}$. Moreover we denote by $a$. $b=a_{i} b_{i}$ the inner product of the vectors $a=\left(a_{i}\right)$ and $b=\left(b_{i}\right)$, by $C e=\left(C_{i j k l} e_{k l}\right)$ the contraction of a fourth order tensor $C=\left(C_{i j k l}\right)$ with a second order tensor $e=\left(e_{k l}\right)$ and by $C e: d=C_{i j k l} e_{k l} d_{i j}$ the inner product of the second order tensors $C e$ and $d=$ $\left(d_{i j}\right)$. Given a function $\theta\left(x^{h}\right)$ defined in $\Omega^{h}$ we denote by $\partial_{i}^{h} \theta$ its partial derivative with respect to $x_{i}^{h}$, that is, $\partial_{i}^{h} \theta=\frac{\partial \theta}{\partial x_{i}^{h}}$.

\subsection{The Three-Dimensional Boundary Value Problem}

In the sequel we consider a static three-dimensional piezoelectric rod model, disregarding the thermal and magnetic effects, and for the case of small deformations and linear piezoelectricity. This model is governed by three groups of equations and boundary conditions, which are described below (cf. Bernadou and Haenel [1], Eringen and Maugin [4], Ikeda [8]):

\section{Mechanical equilibrium equations}

$$
\left[\begin{array}{l}
-\operatorname{div} \sigma^{h}\left(u^{h}, \varphi^{h}\right)=f^{h} \Longleftrightarrow-\partial_{j}^{h} \sigma_{i j}^{h}\left(u^{h}, \varphi^{h}\right)=f_{i}^{h}, \quad \text { in } \Omega^{h}, \\
\sigma^{h}\left(u^{h}, \varphi^{h}\right) v^{h}=g^{h} \Longleftrightarrow \sigma_{i j}^{h}\left(u^{h}, \varphi^{h}\right) v_{j}^{h}=g_{i}^{h}, \quad \text { on } \quad \Gamma_{N}^{h}, \\
u^{h}=0, \quad \text { on } \quad \Gamma_{D}^{h},
\end{array}\right.
$$




\section{Constitutive equations}

$$
\left[\begin{array}{l}
\sigma^{h}\left(u^{h}, \varphi^{h}\right)=C^{h} e^{h}\left(u^{h}\right)-P^{h} E^{h}\left(\varphi^{h}\right) \Longleftrightarrow \sigma_{i j}^{h}=C_{i j k l}^{h} e_{k l}^{h}\left(u^{h}\right)-P_{k i j}^{h} E_{k}^{h}(\varphi), \text { in } \Omega^{h}, \\
D^{h}\left(u^{h}, \varphi^{h}\right)=P^{h} e^{h}\left(u^{h}\right)+\varepsilon^{h} E^{h}\left(\varphi^{h}\right) \Longleftrightarrow D_{k}^{h}=P_{k i j}^{h} e_{i j}^{h}(u)+\varepsilon_{k l}^{h} E_{l}^{h}(\varphi), \text { in } \Omega^{h},
\end{array}\right.
$$

\section{Maxwell-Gauss equations}

$$
\left[\begin{array}{l}
\operatorname{div} D^{h}\left(u^{h}, \varphi^{h}\right)=0 \Longleftrightarrow \partial_{i}^{h} D_{i}^{h}\left(u^{h}, \varphi^{h}\right)=0, \quad \text { in } \quad \Omega^{h}, \\
D^{h}\left(u^{h}, \varphi^{h}\right) v^{h}=0 \Longleftrightarrow D_{i}^{h}\left(u^{h}, \varphi^{h}\right) v_{i}^{h}=0, \quad \text { on } \quad \Gamma_{e N}^{h}, \\
\varphi^{h}=\varphi_{0}^{h}, \quad \text { on } \Gamma_{e D}^{h} .
\end{array}\right.
$$

The unknown of the piezoelectric rod model (4)-(6) is the pair $\left(u^{h}, \varphi^{h}\right)$, where $u^{h}$ : $\Omega^{h} \rightarrow \mathbb{R}^{3}$ denotes the mechanical displacement vector field and $\varphi^{h}: \Omega^{h} \rightarrow \mathbb{R}$ is a scalar field, representing the electric potential. For each point $x^{h} \in \bar{\Omega}^{h}$, the vector $u^{h}\left(x^{h}\right)$ is the mechanical displacement that the point $x^{h}$ undergoes and $\varphi^{h}\left(x^{h}\right)$ is the electric potential at $x^{h}$. The mechanical equilibrium equations express the balance of mechanical loads and internal stresses. We suppose that $f^{h}$ is the density of the applied body forces acting in the rod $\Omega^{h}, g^{h}$ is the density of applied surface forces on $\Gamma_{N}^{h}$ and we assume that the rod is clamped at its extremities $\Gamma_{D}^{h}=\Gamma_{0}^{h} \bigcup \Gamma_{L}^{h}$. The constitutive equations represent the electromechanical interaction that characterizes piezoelectricity. They constitute a relation between the stress tensor $\sigma^{h}: \Omega^{h} \rightarrow \mathbb{R}^{9}$, the electric displacement vector $D^{h}: \Omega^{h} \rightarrow \mathbb{R}^{3}$, the linear strain tensor $e^{h}\left(u^{h}\right)$ and the electric field vector $E^{h}\left(\varphi^{h}\right)$, where

$$
e^{h}\left(u^{h}\right)=\frac{1}{2}\left(\nabla^{h} u^{h}+\left(\nabla^{h} u^{h}\right)^{T}\right) \quad \text { and } \quad E^{h}\left(\varphi^{h}\right)=-\nabla^{h} \varphi^{h},
$$

or, equivalently, componentwise

$$
e_{i j}^{h}\left(u^{h}\right)=\frac{1}{2}\left(\partial_{i}^{h} u_{j}^{h}+\partial_{j}^{h} u_{i}^{h}\right) \quad \text { and } \quad E_{i}^{h}\left(\varphi^{h}\right)=-\partial_{i}^{h} \varphi^{h} .
$$

Moreover $C^{h}=\left(C_{i j k l}^{h}\right)$ is the elastic fourth order tensor field, $P^{h}=\left(P_{i j k}^{h}\right)$ is the piezoelectric third order tensor field, and $\varepsilon^{h}=\left(\varepsilon_{i j}^{h}\right)$ is the dielectric second order tensor field. Finally the Maxwell-Gauss equations are the equations that control the electric displacement vector field $D^{h}$. We assume that $\varphi_{0}^{h}$ is the electric potential applied on $\Gamma_{e D}^{h}$ and there is no electric loading in $\Omega^{h}$ nor on $\Gamma_{e N}^{h}$ (which means the $\operatorname{rod} \Omega^{h}$ is a perfect dielectric body).

In addition we suppose the following hypotheses on the data: The applied forces and applied electric potential have the regularity

$$
f^{h} \in\left[L^{2}\left(\Omega^{h}\right)\right]^{3}, \quad g^{h} \in\left[L^{2}\left(\Gamma_{N}^{h}\right)\right]^{3}, \quad \varphi_{0}^{h} \in H^{\frac{1}{2}}\left(\Gamma_{e D}^{h}\right),
$$

and the three tensor fields $C^{h}=\left(C_{i j k l}^{h}\right), P^{h}=\left(P_{i j k}^{h}\right)$ and $\varepsilon^{h}=\left(\varepsilon_{i j}^{h}\right)$ are independent of $h$ but depend on $x=\left(x_{1}, x_{2}, x_{3}\right) \in \bar{\omega} \times[0, L]$ that is there exist tensor fields $C=\left(C_{i j k l}\right), P=\left(P_{i j k}\right)$ and $\varepsilon=\left(\varepsilon_{i j}\right)$, such that, for any $x^{h}=\left(h x_{1}, h x_{2}, x_{3}\right) \in \bar{\Omega}^{h}$ with 包 Springer 
$\left(x_{1}, x_{2}\right) \in \bar{\omega}$, then

$$
\left[\begin{array}{rl}
C_{i j k l}^{h}\left(x^{h}\right) & =C_{i j k l}(x), \\
P_{i j k}^{h}\left(x^{h}\right) & =P_{i j k}(x), \\
\varepsilon_{i j}^{h}\left(x^{h}\right) & =\varepsilon_{i j}(x),
\end{array} \quad \text { and } \quad x=\left(x_{1}, x_{2}, x_{3}\right) \in \bar{\omega} \times[0, L],\right.
$$

where $C_{i j k l}, P_{i j k}$ and $\varepsilon_{i j}$ are independent of $h$. We also assume that $C_{i j k l}, P_{i j k}, \varepsilon_{i j}$ are smooth enough functions defined in $\bar{\omega} \times[0, L]$ that satisfy the following symmetric and positivity properties:

- $\quad$ in $\bar{\omega} \times[0, L]$

$$
\begin{aligned}
C_{i j k l} & =C_{j i k l}=C_{k l i j}, & C_{\alpha \beta \gamma 3} & =0=C_{\alpha 333}, \\
P_{i j k} & =P_{i k j}, & \varepsilon_{i j} & =\varepsilon_{j i},
\end{aligned}
$$

- there exists constants $c_{1}>0$ and $c_{2}>0$, such that

$$
C_{i j k l}(x) M_{k l} M_{i j} \geq c_{1} \sum_{i, j=1}^{3}\left(M_{i j}\right)^{2} \quad \text { and } \quad \varepsilon_{i j}(x) \theta_{i} \theta_{j} \geq c_{2} \sum_{i=1}^{3} \theta_{i}^{2}
$$

for every symmetric $3 \times 3$ real matrix $M$ and any vector $\theta \in \mathbb{R}^{3}$, and for every $x \in \bar{\omega} \times[0, L]$.

The hypotheses $C_{\alpha \beta \gamma 3}=0=C_{\alpha 333}$ mean that the rod has elastic symmetry with respect to the plane $x_{3}=0$ (cf. Green and Zerna [5]). Consequently the material is monoclinic in the plane $O X_{1} X_{2}$ and the number of independent elastic coefficients $\left(C_{i j k l}\right)$ is equal to 13 .

Due the elastic properties (11), the components of the elasticity tensor $C$ can be identified with the following matrix $\mathcal{C}$

$$
\mathcal{C}=\left[\begin{array}{cccccc}
C_{1111} & C_{1112} & C_{1122} & 0 & 0 & C_{1133} \\
C_{1211} & C_{1212} & C_{1222} & 0 & 0 & C_{1233} \\
C_{2211} & C_{2212} & C_{2222} & 0 & 0 & C_{2233} \\
0 & 0 & 0 & C_{1313} & C_{1323} & 0 \\
0 & 0 & 0 & C_{2313} & C_{2323} & 0 \\
C_{3311} & C_{3312} & C_{3322} & 0 & 0 & C_{3333}
\end{array}\right]
$$

The first positivity hypothesis in (12) guarantees that the matrix $\mathcal{C}$ is positive definite, consequently, $C_{3333}>0$, and the sub-matrices

$$
\mathcal{M}=\left[\begin{array}{lll}
C_{1111} & C_{1112} & C_{1122} \\
C_{1211} & C_{1212} & C_{1222} \\
C_{2211} & C_{2212} & C_{2222}
\end{array}\right] \quad \text { and } \quad \mathcal{N}=\left[\begin{array}{ll}
C_{1313} & C_{1323} \\
C_{2313} & C_{2323}
\end{array}\right]
$$

are also positive definite. In particular, for a homogeneous and isotropic material, the elasticity coefficients $C_{i j k l}$ are constants, defined by,

$$
C_{i j k l}=\lambda \delta_{i j} \delta_{k l}+\mu\left(\delta_{i k} \delta_{j l}+\delta_{i l} \delta_{j k}\right),
$$


where $\lambda$ and $\mu$ are the Lamé constants, $\delta_{i j}=0$ if $i \neq j, \delta_{i j}=1$ if $i=j$, and the matrix $\mathcal{C}$ in (13) becomes

$$
\mathcal{C}=\left[\begin{array}{cccccc}
\lambda+2 \mu & 0 & \lambda & 0 & 0 & \lambda \\
0 & \mu & 0 & 0 & 0 & 0 \\
\lambda & 0 & \lambda+2 \mu & 0 & 0 & \lambda \\
0 & 0 & 0 & \mu & 0 & 0 \\
0 & 0 & 0 & 0 & \mu & 0 \\
\lambda & 0 & \lambda & 0 & 0 & \lambda+2 \mu
\end{array}\right]
$$

\subsection{Variational Formulation of the Three-Dimensional Rod Model}

We define the space of admissible displacements

$$
V^{h}=\left\{v^{h} \in\left[H^{1}\left(\Omega^{h}\right)\right]^{3}: \quad v_{\mid \Gamma_{D}^{h}}^{h}=0\right\}
$$

with the norm $\left\|v^{h}\right\|_{V^{h}}=\left\|e^{h}\left(v^{h}\right)\right\|_{\left[L^{2}\left(\Omega^{h}\right)\right]^{9}}$, and the space of admissible electric potentials

$$
\Psi^{h}=\left\{\psi^{h} \in H^{1}\left(\Omega^{h}\right): \quad \psi_{\mid \Gamma_{e D}^{h}}^{h}=0\right\}
$$

with the norm $\left\|\psi^{h}\right\|_{\Psi^{h}}=\left\|\nabla \psi^{h}\right\|_{\left[L^{2}\left(\Omega^{h}\right)\right]^{3}}$. Let $\bar{\varphi}^{h}=\varphi^{h}-\varphi_{0}^{h}$, where $\varphi_{0}^{h}$ is a trace lifting in $H^{1}\left(\Omega^{h}\right)$ of the applied potential acting on $\Gamma_{e D}^{h}$ (cf. (6)). In the sequel we denote by $\varphi_{0}^{h}$ the trace lifting as well as its boundary value at $\Gamma_{e D}^{h}$. Then, the variational formulation of the system (4)-(6) is defined by

$$
\left\{\begin{array}{l}
\text { Find } \quad\left(u^{h}, \bar{\varphi}^{h}\right) \in V^{h} \times \Psi^{h} \quad \text { such that: } \\
a^{h}\left(\left(u^{h}, \bar{\varphi}^{h}\right),\left(v^{h}, \psi^{h}\right)\right)=l^{h}\left(v^{h}, \psi^{h}\right), \quad \forall\left(v^{h}, \psi^{h}\right) \in V^{h} \times \Psi^{h},
\end{array}\right.
$$

where

$$
\left[\begin{array}{l}
a^{h}\left(\left(u^{h}, \bar{\varphi}^{h}\right),\left(v^{h}, \psi^{h}\right)\right) \\
=\int_{\Omega^{h}} C e^{h}\left(u^{h}\right): e^{h}\left(v^{h}\right) d x^{h}+\int_{\Omega^{h}} \varepsilon_{i j} \partial_{i}^{h} \bar{\varphi}^{h} \partial_{j}^{h} \psi^{h} d x^{h} \\
+\int_{\Omega^{h}} P_{i j k}\left(\partial_{i}^{h} \bar{\varphi}^{h} e_{j k}^{h}\left(v^{h}\right)-\partial_{i}^{h} \psi^{h} e_{j k}^{h}\left(u^{h}\right)\right) d x^{h}
\end{array}\right.
$$

and

$$
\left[\begin{array}{l}
l^{h}\left(v^{h}, \psi^{h}\right)=\int_{\Omega^{h}} f^{h} \cdot v^{h} d x^{h}+\int_{\Gamma_{N}^{h}} g^{h} \cdot v^{h} d \Gamma_{N}^{h} \\
-\int_{\Omega^{h}} \varepsilon_{i j} \partial_{i}^{h} \varphi_{0}^{h} \partial_{j}^{h} \psi^{h} d x^{h}-\int_{\Omega^{h}} P_{i j k} \partial_{i}^{h} \varphi_{0}^{h} e_{j k}^{h}\left(v^{h}\right) d x^{h} .
\end{array}\right.
$$

To obtain (19) we first do the inner product of the first equation of (4) by $v^{h} \in V^{h}$ and we multiply the first equation of (6) by $\psi^{h} \in \Psi^{h}$; afterwards adding the two 照 Springer 
resulting equations, integrating in $\Omega^{h}$, applying the Green's formula, and using all the boundary conditions and the constitutive equations defined in (4)-(6) we get (19).

The definition of $a^{h}(.$, .) clearly implies that

$$
\left[\begin{array}{l}
a^{h}\left(\left(v^{h}, \psi^{h}\right),\left(v^{h}, \psi^{h}\right)\right) \\
=\int_{\Omega^{h}} C e^{h}\left(v^{h}\right): e^{h}\left(v^{h}\right) d x^{h}+\int_{\Omega^{h}} \varepsilon_{i j} \partial_{i}^{h} \psi^{h} \partial_{j}^{h} \psi^{h} d x^{h},
\end{array}\right.
$$

and therefore, by the positivity properties (12), the bilinear form $a^{h}(.,$.$) is coercive.$ Consequently, by the Lax-Milgram lemma, the variational problem (19) has a unique solution.

\section{Asymptotic Analysis}

In this section we apply the asymptotic analysis procedure (as developed for elastic plates by Ciarlet [2], and for elastic rods by Trabucho and Viaño [13]) to the variational problem (19). We first transform the three-dimensional piezoelectric rod problem (19), into an equivalent problem depending on $h$, but posed over the scaled $\operatorname{rod} \Omega=\omega \times(0, L)$ independent of $h$ (we use appropriate scalings of the unknowns $u^{h}, \varphi^{h}$ and convenient assumptions on the data). Then, we study the behavior of the scaled displacements, electric potentials, stresses and electric vectors as $h \rightarrow 0^{+}$. The Theorems 3.1 and 3.2 prove the existence of the weak limits. The Theorem 3.3 characterizes the limit model.

\subsection{The Scaled Three-Dimensional Rod Model}

We redefine here the three-dimensional variational problem (19) in the domain $\Omega=$ $\omega \times(0, L)$ independent of $h$. Each $x=\left(x_{1}, x_{2}, x_{3}\right) \in \Omega$ is associated with the element $x^{h}=\left(h x_{1}, h x_{2}, x_{3}\right) \in \Omega^{h}$, and we also consider the subsets defined in (1)-(2) for the choice $h=1$, that is

$$
\begin{aligned}
& \Omega=\omega \times(0, L), \quad \Gamma_{0}=\omega \times\{0\}, \quad \Gamma_{L}=\omega \times\{L\}, \\
& \Gamma_{D}=\Gamma_{0} \bigcup \Gamma_{L}, \quad \Gamma_{N}=\gamma \times(0, L), \quad \gamma=\partial \omega, \\
& \Gamma_{e D}=\gamma_{e} \times(0, L), \quad \Gamma_{e N}=\partial \Omega \backslash \Gamma_{e D} .
\end{aligned}
$$

We denote by $v=\left(v_{1}, v_{2}\right)=\left(v_{\alpha}\right)$ the unit outer normal vector along $\partial \omega$, and by $\partial_{i}=$ $\frac{\partial}{\partial x_{i}}$ and $\partial_{i j}=\frac{\partial^{2}}{\partial x_{i} \partial x_{j}}$, the first and second partial derivatives with respect to $x_{i}$ and $x_{j}$.

We suppose that the data satisfy the hypotheses

$$
\begin{array}{ll}
f_{\alpha}^{h}\left(x^{h}\right)=h f_{\alpha}(x), & f_{3}^{h}\left(x^{h}\right)=f_{3}(x), \quad x \in \Omega, \\
g_{\alpha}^{h}\left(x^{h}\right)=h^{2} g_{\alpha}(x), & g_{3}^{h}\left(x^{h}\right)=h g_{3}(x), \quad x \in \Gamma_{N}, \\
\varphi_{0}^{h}\left(x^{h}\right)=h \varphi_{0}(x), \quad x \in \bar{\Omega}, &
\end{array}
$$


where $f_{i} \in L^{2}(\Omega), g_{i} \in L^{2}\left(\Gamma_{N}\right)$ and $\varphi_{0} \in H^{1}(\Omega)$. For the unknowns, $u^{h} \in V^{h}$ and $\varphi^{h} \in \Psi^{h}$, and for any admissible displacement or electric potential, respectively, $v^{h} \in V^{h}$ and $\psi^{h} \in \Psi^{h}$, the following scalings are assumed:

$$
\begin{aligned}
u_{\alpha}^{h}\left(x^{h}\right) & =h^{-1} u_{\alpha}(h)(x), \quad u_{3}^{h}\left(x^{h}\right)=u_{3}(h)(x), \quad x \in \Omega, \\
v_{\alpha}^{h}\left(x^{h}\right) & =h^{-1} v_{\alpha}(h)(x), \quad v_{3}^{h}\left(x^{h}\right)=v_{3}(h)(x), \quad x \in \Omega, \\
\varphi^{h}\left(x^{h}\right) & =h \varphi(h)(x), \quad x \in \Omega, \\
\psi^{h}\left(x^{h}\right) & =h \psi(h)(x), \quad x \in \Omega .
\end{aligned}
$$

We remark that the assumptions and the scalings indicated in (24)-(25) for the mechanical forces and the displacements correspond exactly to the contravariant and the covariant transformations of the components of the mechanical forces and the displacements, respectively, and which are associated with the coordinate change $\left(x_{1}, x_{2}, x_{3}\right) \in \Omega \rightarrow x^{h}=\left(h x_{1}, h x_{2}, x_{3}\right) \in \Omega^{h}$ (cf. [14], p. 439). Moreover these assumptions and scalings are the same as in Trabucho and Viaño [13], pages 516-517 and Remark 2.1. The scaling orders of $\varphi_{0}^{h}$ and $\varphi^{h}$ (as well as of $\psi^{h}$ ) are chosen so that an asymptotic solution exists for the variational problem (32), which means that these are selected in such a way that we are able to compute the limit of the scaled three-dimensional rod model (32) as $h \rightarrow 0$. We also note that a similar reasoning is used for example in Rahmoune et al. [10] and Sene [12].

The vector fields $u(h)$ and $v(h)$ are in the scaled admissible displacement space $V$, and the electric potentials $\varphi(h)$ and $\psi(h)$ belong to the scaled admissible electric potential space $\Psi$, where $V$ and $\Psi$ are defined by

$$
\begin{array}{ll}
V=\left\{v \in\left[H^{1}(\Omega)\right]^{3}:\right. & \left.v_{\mid \Gamma_{D}}=0\right\}, \\
\Psi=\left\{\psi \in H^{1}(\Omega):\right. & \left.\psi_{\mid \Gamma_{e D}}=0\right\} .
\end{array}
$$

The first space is equipped with the norm $\|v\|_{V}=\|e(v)\|_{\left(L^{2}(\Omega)\right)^{9}}$ and the second with the norm $\|\psi\|_{\Psi}=\|\nabla \psi\|_{\left(L^{2}(\Omega)\right)^{3}}$.

For any $v \in V$ the second order symmetric tensor field $\kappa(h)(v)=\left(\kappa_{i j}(h)(v)\right)$ is defined by

$$
\begin{aligned}
\kappa_{\alpha \beta}(h)(v) & =\frac{1}{h^{2}} e_{\alpha \beta}(v)=\frac{1}{2 h^{2}}\left(\partial_{\beta} v_{\alpha}+\partial_{\alpha} v_{\beta}\right), \\
\kappa_{\alpha 3}(h)(v) & =\frac{1}{h} e_{\alpha 3}(v)=\frac{1}{2 h}\left(\partial_{3} v_{\alpha}+\partial_{\alpha} v_{3}\right), \\
\kappa_{33}(h)(v) & =e_{33}(v)=\partial_{3} v_{3} .
\end{aligned}
$$

In particular, when $v=u(h)$ we set $\kappa(h)=\kappa(h)(u(h))$. As a consequence of the scalings (25) and (27) we have for any $v^{h}$ in $V^{h}$

$$
\begin{aligned}
& e_{\alpha \beta}^{h}\left(v^{h}\right)=h^{-2} e_{\alpha \beta}(v(h))=\kappa_{\alpha \beta}(h)(v(h)), \\
& e_{\alpha 3}^{h}\left(v^{h}\right)=h^{-1} e_{\alpha 3}(v(h))=\kappa_{\alpha 3}(h)(v(h)), \\
& e_{33}^{h}\left(v^{h}\right)=e_{33}(v(h))=\kappa_{33}(h)(v(h)),
\end{aligned}
$$

therefore

$$
e^{h}\left(u^{h}\right)=\kappa(h)(u(h))=\kappa(h) \quad \text { and } \quad e^{h}\left(v^{h}\right)=\kappa(h)(v(h)) .
$$


The scalings of the stress tensor $\sigma^{h}$, the electric displacement vector $D^{h}$ and the electric vector $E^{h}$ are induced by (25) and are defined by

$$
\begin{aligned}
& \sigma_{i j}(h)(u(h), \varphi(h))=\sigma_{i j}^{h}\left(u^{h}, \varphi^{h}\right), \quad D_{i}(u(h), \varphi(h))=D_{i}^{h}\left(u^{h}, \varphi^{h}\right), \\
& E_{i}(\varphi(h))=E_{i}^{h}\left(\varphi^{h}\right),
\end{aligned}
$$

where

$$
\begin{aligned}
\sigma_{i j}^{h}\left(u^{h}, \varphi^{h}\right) & =C_{i j m l} \kappa_{m l}(h)-P_{m i j} E_{m}(h)(\varphi(h))=\sigma_{i j}(h)(u(h), \varphi(h)), \\
D_{i}^{h}\left(u^{h}, \varphi^{h}\right) & =P_{i l m} \kappa_{l m}(h)+\varepsilon_{i j} E_{j}(h)=D_{i}(h)(u(h), \varphi(h)), \\
E_{\alpha}(h)(\varphi(h)) & =-\partial_{\alpha} \varphi(h), \quad E_{3}(h)(\varphi(h))=-h \partial_{3} \varphi(h)
\end{aligned}
$$

Using all these transformations and assumptions on the data, a straightforward computation shows that (19) is equivalent to the following scaled three-dimensional variational rod model

$$
\left\{\begin{array}{l}
\text { Find } \quad(u(h), \bar{\varphi}(h)) \in V \times \Psi \quad \text { such that: } \\
a(h)((u(h), \bar{\varphi}(h)),(v, \psi))=l(h)(v, \psi), \quad \forall(v, \psi) \in V \times \Psi
\end{array}\right.
$$

where

$$
\left[\begin{array}{l}
a(h)((u(h), \bar{\varphi}(h)),(v, \psi))=\int_{\Omega} C \kappa(h): \kappa(h)(v) d x+\int_{\Omega} \varepsilon_{\alpha \beta} \partial_{\alpha} \bar{\varphi}(h) \partial_{\beta} \psi d x \\
+\int_{\Omega} P_{\alpha i j}\left[\partial_{\alpha} \bar{\varphi}(h) \kappa_{i j}(h)(v)-\partial_{\alpha} \psi \kappa_{i j}(h)\right] d x \\
+h \int_{\Omega} \varepsilon_{3 \alpha}\left[\partial_{\alpha} \bar{\varphi}(h) \partial_{3} \psi+\partial_{3} \bar{\varphi}(h) \partial_{\alpha} \psi\right] d x \\
+h \int_{\Omega} P_{3 i j}\left[\partial_{3} \bar{\varphi}(h) \kappa_{i j}(h)(v)-\partial_{3} \psi \kappa_{i j}(h)\right] d x+h^{2} \int_{\Omega} \varepsilon_{33} \partial_{3} \bar{\varphi}(h) \partial_{3} \psi d x,
\end{array}\right.
$$

and

$$
\left[\begin{array}{l}
l(h)(v, \psi)=\int_{\Omega} f \cdot v d x+\int_{\Gamma_{N}} g \cdot v d \Gamma_{N}-\int_{\Omega} \varepsilon_{\alpha \beta} \partial_{\alpha} \varphi_{0} \partial_{\beta} \psi d x \\
-h \int_{\Omega} \varepsilon_{\alpha 3}\left[\partial_{\alpha} \varphi_{0} \partial_{3} \psi+\partial_{3} \varphi_{0} \partial_{\alpha} \psi\right] d x-h^{2} \int_{\Omega} \varepsilon_{33} \partial_{3} \varphi_{0} \partial_{3} \psi d x \\
-h \int_{\Omega} P_{3 i j} \partial_{3} \varphi_{0} \kappa_{i j}(h)(v) d x-\int_{\Omega} P_{\alpha i j} \partial_{\alpha} \varphi_{0} \kappa_{i j}(h)(v) d x .
\end{array}\right.
$$

\subsection{Computation of a Weak Limit}

Here we essentially compute and identify a weak limit of the scaled displacements and electric potentials $(u(h), \varphi(h))$ when $h \rightarrow 0^{+}$. 


\subsubsection{Functional Spaces}

In order to compute the limits it is necessary to introduce the spaces $V_{B N}, \Psi_{l}$ and $\Psi_{l 0}$. Let

$$
\begin{aligned}
V_{B N} & =\left\{v \in V: \quad e_{\alpha \beta}(v)=e_{3 \beta}(v)=0\right\}, \\
\Psi_{l} & =\left\{\psi \in L^{2}(\Omega): \quad \partial_{\alpha} \psi \in L^{2}(\Omega), \quad \alpha=1,2\right\}, \\
\Psi_{l 0} & =\left\{\psi \in \Psi_{l}: \quad \psi_{\mid \Gamma_{e D}}=0\right\} .
\end{aligned}
$$

The space $V_{B N}$, which is called the Bernoulli-Navier mechanical displacement space, is equivalently defined by

$$
\begin{gathered}
V_{B N}=\left\{v=\left(v_{1}, v_{2}, v_{3}\right) \in V: \quad v_{\alpha}\left(x_{1}, x_{2}, x_{3}\right)=\eta_{\alpha}\left(x_{3}\right), \quad \eta_{\alpha} \in H_{0}^{2}(] 0, L[),\right. \\
\left.\left.\left.v_{3}\left(x_{1}, x_{2}, x_{3}\right)=\eta_{3}\left(x_{3}\right)-x_{\alpha} \partial_{3} \eta_{\alpha}\left(x_{3}\right), \quad \eta_{3} \in H_{0}^{1}(] 0, L\right]\right)\right\},
\end{gathered}
$$

and it is equipped with the norm $\|\cdot\|_{V_{N B}}$, where

$$
\begin{aligned}
\|v\|_{V_{N B}}^{2} & =\left\|e_{33}(v)\right\|_{L^{2}(\Omega)}^{2}=\int_{\Omega}\left|\partial_{3} \eta_{3}-x_{\alpha} \partial_{33} \eta_{\alpha}\right|^{2} d x \\
& =\operatorname{meas}(\omega)\left\|\partial_{3} \eta_{3}\right\|_{L^{2}(10, L D}^{2}+\left(\int_{\omega} x_{\alpha}^{2} d \omega\right)\left\|\partial_{33} \eta_{\alpha}\right\|_{\left.\left.L^{2}(] 0, L\right]\right)}^{2} .
\end{aligned}
$$

The space $\Psi_{l}$ is equipped with the norm $\|\cdot\|_{\Psi_{l}}$ defined by

$$
\|\varphi\|_{\Psi_{l}}=\left(\|\varphi\|_{L^{2}(\Omega)}^{2}+\sum_{\alpha=1}^{2}\left\|\partial_{\alpha} \varphi\right\|_{L^{2}(\Omega)}^{2}\right)^{\frac{1}{2}} .
$$

We observe that, for any $\psi \in \Psi_{l}$ the trace $\psi_{\mid \Gamma_{e D}}$ is well defined. In fact, for any $\psi \in \Psi_{l}$, and for any fixed $x_{3} \in(0, L)$, we can define $\chi\left(x_{1}, x_{2}\right)=\psi\left(x_{1}, x_{2}, x_{3}\right)$ and $\chi \in$ $H^{1}(\omega)$; the trace of $\chi$ is an element of $H^{\frac{1}{2}}\left(\gamma_{e}\right)$, that satisfies $\left\|\chi_{\mid \gamma_{e}}\right\|_{L^{2}\left(\gamma_{e}\right)} \leq c\|\chi\|_{H^{1}(\omega)}$ with $c$ a constant, that does not depend on the fixed $x_{3}$, therefore integrating the previous inequality in $x_{3}$, from 0 to $L$, we get $\left\|\psi_{\mid \Gamma_{e D}}\right\|_{L^{2}\left(\Gamma_{e D}\right)}^{2} \leq\|\psi\|_{\Psi_{l}}^{2}$. This latter inequality proves that $\psi_{\mid \Gamma_{e D}}$ is well defined and the trace operator acting from $\Psi_{l}$ to $L^{2}\left(\Gamma_{e D}\right)$ is continuous. Moreover, the function $\|\cdot\|_{\Psi_{l 0}}$ defined by

$$
\|\psi\|_{\Psi_{l 0}}=\left(\sum_{\alpha=1}^{2}\left\|\partial_{\alpha} \psi\right\|_{L^{2}(\Omega)}^{2}\right)^{\frac{1}{2}}
$$

is a norm equivalent to the norm induced in $\Psi_{l 0}$ by the norm of $\Psi_{l}$. In addition, the following result can also be established.

PROPOSITION 3.1. The space $D(\Omega)$ is dense in $\Psi_{l 0}$ equipped with the norm $\|.\|_{\Psi_{l}}$.

Proof. Similar to Proposition 3.2 of Sene [12].

\subsubsection{Weak Limits and Variational Limit Rod Model}

The weak convergence is proved in the following theorem: 
THEOREM 3.1. There exist $u \in\left[H^{1}(\Omega)\right]^{3}, \kappa \in\left[L^{2}(\Omega)\right]^{9}$ and $\varphi \in L^{2}(\Omega)$ and subsequences $\{u(h)\}_{h>0},\{\kappa(h)\}_{h>0}$ and $\left\{\left(\partial_{1} \varphi(h), \partial_{2} \varphi(h), h \partial_{3} \varphi(h)\right)\right\}_{h>0}$ (still indexed by $h$ ), such that the following weak convergences are satisfied, when $h \rightarrow 0^{+}$,

$$
\begin{aligned}
& u(h) \rightarrow u \text { in }\left[H^{1}(\Omega)\right]^{3}, \\
& \kappa(h) \rightarrow \kappa \text { in }\left[L^{2}(\Omega)\right]^{9}, \\
& \varphi(h) \rightarrow \varphi \text { in } L^{2}(\Omega), \\
& \left(\partial_{1} \varphi(h), \partial_{2} \varphi(h), h \partial_{3} \varphi(h)\right) \rightarrow\left(\partial_{1} \varphi, \partial_{2} \varphi, 0\right) \quad \text { in }\left[L^{2}(\Omega)\right]^{3} .
\end{aligned}
$$

Moreover, the limits $u, \kappa$ and $\varphi$ belong to the spaces $V_{B N},\left(L^{2}(\Omega)\right)^{9}$ and $\Psi_{l}$, respectively, $\varphi=\varphi_{0}$ on $\Gamma_{e D}$, and there exists an implicit function $\theta=\left(\theta_{1}, \theta_{2}, \theta_{3}\right)$ of $u$, $\varphi$ and $\kappa$ (defined in Equations (50) and (53)) and that satisfies $\theta_{i}\left(., ., x_{3}\right) \in H^{1}(\omega)$ almost everywhere in $(0, L)$, for $i=1,2,3$, such that

$$
\kappa_{11}=\frac{-\operatorname{det}\left[\begin{array}{lll}
C_{3311} e_{33}(u)+P_{\alpha 11} \partial_{\alpha} \varphi-\partial_{2} \theta_{1} & C_{1211} & C_{2211} \\
C_{3312} e_{33}(u)+P_{\alpha 12} \partial_{\alpha} \varphi-\partial_{2} \theta_{2} & C_{1212} & C_{2212} \\
C_{3322} e_{33}(u)+P_{\alpha 22} \partial_{\alpha} \varphi+\partial_{1} \theta_{2} & C_{1222} & C_{2222}
\end{array}\right]}{\operatorname{det} \mathcal{M}},
$$

$$
\kappa_{12}=\frac{-\operatorname{det}\left[\begin{array}{lll}
C_{1111} & C_{3311} e_{33}(u)+P_{\alpha 11} \partial_{\alpha} \varphi-\partial_{2} \theta_{1} & C_{2211} \\
C_{1112} & C_{3312} e_{33}(u)+P_{\alpha 12} \partial_{\alpha} \varphi-\partial_{2} \theta_{2} & C_{2212} \\
C_{1122} & C_{3322} e_{33}(u)+P_{\alpha 22} \partial_{\alpha} \varphi+\partial_{1} \theta_{2} & C_{2222}
\end{array}\right]}{2 \operatorname{det} \mathcal{M}},
$$

$$
\kappa_{22}=\frac{-\operatorname{det}\left[\begin{array}{lll}
C_{1111} & C_{1211} & C_{3311} e_{33}(u)+P_{\alpha 11} \partial_{\alpha} \varphi-\partial_{2} \theta_{1} \\
C_{1112} & C_{1212} & C_{3312} e_{33}(u)+P_{\alpha 12} \partial_{\alpha} \varphi-\partial_{2} \theta_{2} \\
C_{1122} & C_{1222} & C_{3322} e_{33}(u)+P_{\alpha 22} \partial_{\alpha} \varphi+\partial_{1} \theta_{2}
\end{array}\right]}{\operatorname{det} \mathcal{M}},
$$$$
\kappa_{13}=\frac{\operatorname{det}\left[\begin{array}{cc}
-\frac{1}{2} P_{\alpha 31} \partial_{\alpha} \varphi+\frac{1}{2} \partial_{2} \theta_{3} & C_{2331} \\
-\frac{1}{2} P_{\alpha 32} \partial_{\alpha} \varphi-\frac{1}{2} \partial_{1} \theta_{3} & C_{2332}
\end{array}\right]}{\operatorname{det} \mathcal{N}},
$$$$
\kappa_{32}=\frac{\operatorname{det}\left[\begin{array}{c}
C_{1331}-\frac{1}{2} P_{\alpha 31} \partial_{\alpha} \varphi+\frac{1}{2} \partial_{2} \theta_{3} \\
C_{1332}-\frac{1}{2} P_{\alpha 32} \partial_{\alpha} \varphi-\frac{1}{2} \partial_{1} \theta_{3}
\end{array}\right]}{\operatorname{det} \mathcal{N}},
$$

$$
\kappa_{33}=e_{33}(u)
$$

Proof. Taking $(v, \psi)=(u(h), \bar{\varphi}(h))$ in (32) we obtain

$$
\left\{\begin{array}{l}
\|u(h)\|_{V}^{2}+\int_{\Omega} \kappa(h): \kappa(h) d x \\
+\left\|\partial_{1} \varphi(h)\right\|_{L^{2}(\Omega)}^{2}+\left\|\partial_{2} \varphi(h)\right\|_{L^{2}(\Omega)}^{2}+\left\|h \partial_{3} \varphi(h)\right\|_{L^{2}(\Omega)}^{2}<b,
\end{array}\right.
$$


where $b>0$ is a constant independent of $h$. Using the Poincaré-Friedrichs inequality, for any $x_{3}$ fixed in $(0, L)$, we have

$$
\left\{\begin{array}{l}
\int_{\omega}\left|\bar{\varphi}(h)\left(x_{1}, x_{2}, x_{3}\right)\right|^{2} d \omega \leq \\
\leq b\left(\int_{\omega}\left|\partial_{1} \bar{\varphi}(h)\left(x_{1}, x_{2}, x_{3}\right)\right|^{2} d \omega+\int_{\omega}\left|\partial_{2} \bar{\varphi}(h)\left(x_{1}, x_{2}, x_{3}\right)\right|^{2} d \omega\right),
\end{array}\right.
$$

where $b$ is another constant which does not depend on $x_{3}$. Thus, integrating the previous inequality, in $x_{3}$, from 0 to $L$, and using (47) we conclude that $\bar{\varphi}(h)$ is bounded in $L^{2}(\Omega)$. Therefore, arguing as in Sene [12] (proposition 3.1) we have the weak convergence indicated in (40). We remark that the weak limit $\bar{\varphi}$ of the sequence $\bar{\varphi}(h)$ belongs to $\Psi$; hence the weak limit $\varphi$ of the sequence $\varphi(h)$ satisfies $\varphi_{\mid \Gamma_{e D}}=\bar{\varphi}_{\mid \Gamma_{e D}}+\varphi_{0 \mid \Gamma_{e D}}=\varphi_{0 \mid \Gamma_{e D}}$.

Since $u(h)$ converges weakly to $u$ in $\left[H^{1}(\Omega)\right]^{3}$ and $u(h) \in V$, then also $u \in V$. In addition, this weak convergence implies that $e_{i j}(u(h))$ converges weakly to $e_{i j}(u)$ in $\left[L^{2}(\Omega)\right]$. On the other hand, the inequality (47) assures that $\kappa_{i j}(h)$ is bounded in $L^{2}(\Omega)$. Consequently, the sequences $e_{\alpha 3}(u(h))=h \kappa_{\alpha 3}(h)(u(h))$ and $e_{\alpha \beta}(u(h))=$ $h^{2} \kappa_{\alpha \beta}(h)(u(h))$ converge strongly to zero in $L^{2}(\Omega)$, hence $e_{\alpha \beta}(u)=0=e_{\alpha 3}(u)$. Therefore $u \in V_{B N}$.

To obtain (41)-(43) we multiply (32) by $h^{2}$, consider $\psi=0$ and take the limit, when $h \rightarrow 0^{+}$and we have

$$
\int_{\Omega}\left(C_{i j \beta \rho} \kappa_{i j}+P_{\alpha \beta \rho} \partial_{\alpha}\left(\bar{\varphi}+\varphi_{0}\right)\right) e_{\beta \rho}(v) d x=0, \quad \forall v \in V
$$

Applying theorem 3.1 of [6] to this latter variational equation we have in $\Omega$

$$
\begin{aligned}
C_{i j 11} \kappa_{i j}+P_{\alpha 11} \partial_{\alpha}\left(\bar{\varphi}+\varphi_{0}\right) & =+\partial_{2} \theta_{1}, \\
C_{i j 21} \kappa_{i j}+P_{\alpha 21} \partial_{\alpha}\left(\bar{\varphi}+\varphi_{0}\right) & =-\partial_{1} \theta_{1}, \\
C_{i j 12} \kappa_{i j}+P_{\alpha 12} \partial_{\alpha}\left(\bar{\varphi}+\varphi_{0}\right) & =+\partial_{2} \theta_{2}, \\
C_{i j 22} \kappa_{i j}+P_{\alpha 22} \partial_{\alpha}\left(\bar{\varphi}+\varphi_{0}\right) & =-\partial_{1} \theta_{2}, \\
\text { with }-\partial_{1} \theta_{1} & =\partial_{2} \theta_{2},
\end{aligned}
$$

where $\theta_{\alpha}\left(., ., x_{3}\right) \in H^{1}(\omega)$ almost everywhere in $(0, L)$; the last equation is due to the symmetry of $C_{i j k l}, P_{i j k}$ and $\kappa_{i j}$.

Remarking that $\varphi=\bar{\varphi}+\varphi_{0}$ and using the symmetry properties of $C_{i j k l}$ indicated in (11), we easily check that the components $\kappa_{11}, \kappa_{12}$ and $\kappa_{22}$ are the solution of the following system, which is equivalent to (50):

$$
\mathcal{M}\left[\begin{array}{c}
k_{11} \\
2 k_{12} \\
k_{22}
\end{array}\right]=\left[\begin{array}{l}
-C_{3311} e_{33}(u)-P_{\alpha 11} \partial_{\alpha} \varphi+\partial_{2} \theta_{1} \\
-C_{3312} e_{33}(u)-P_{\alpha 12} \partial_{\alpha} \varphi+\partial_{2} \theta_{2} \\
-C_{3322} e_{33}(u)-P_{\alpha 22} \partial_{\alpha} \varphi-\partial_{1} \theta_{2}
\end{array}\right]
$$

Consequently, we have (41)-(43).

典 Springer 
To justify the formulas for the components $\kappa_{13}$ and $\kappa_{23}$ we can follow an analogous procedure. If we multiply (32) by $h$, take $\psi=0$ and $v_{\alpha}=0$, for $\alpha=1,2$, and compute the limit when $h \rightarrow 0^{+}$, we obtain

$$
\int_{\Omega}\left(C_{i j 3 \beta} \kappa_{i j}+P_{\alpha 3 \beta} \partial_{\alpha}\left(\bar{\varphi}+\varphi_{0}\right)\right) \partial_{\beta} v_{3} d x=0, \quad \forall v_{3} \in H^{1}(\Omega), \quad v_{3 \mid \Gamma_{D}}=0
$$

Applying again Theorem 3.1 of [6], we have in $\Omega$

$$
\begin{aligned}
& C_{i j 31} \kappa_{i j}+P_{\alpha 31} \partial_{\alpha}\left(\bar{\varphi}+\varphi_{0}\right)=+\partial_{2} \theta_{3}, \\
& C_{i j 32} \kappa_{i j}+P_{\alpha 32} \partial_{\alpha}\left(\bar{\varphi}+\varphi_{0}\right)=-\partial_{1} \theta_{3},
\end{aligned}
$$

where $\theta_{3}\left(., ., x_{3}\right) \in H^{1}(\omega)$ almost everywhere in $(0, L)$. Using once more the properties (11), we conclude that (53) is equivalent to

$$
\mathcal{N}\left[\begin{array}{l}
k_{13} \\
k_{23}
\end{array}\right]=\left[\begin{array}{c}
-\frac{1}{2} P_{\alpha 31} \partial_{\alpha} \varphi+\frac{1}{2} \partial_{2} \theta_{3} \\
-\frac{1}{2} P_{\alpha 32} \partial_{\alpha} \varphi-\frac{1}{2} \partial_{1} \theta_{3}
\end{array}\right]
$$

which gives (44)-(45). Finally, the formula (46) results from the weak convergence $e_{i j}(u(h)) \rightarrow e_{i j}(u)$ and the fact that $\kappa_{33}(u(h))=e_{33}(u(h))$.

The weak convergence results (40) imply the following theorem, which expresses the limit stress tensor $\sigma$ and the limit electric displacement vector $D$ as functions of the limit mechanical displacement $u$ and of the limit electric potential $\varphi$.

THEOREM 3.2. There exist $\sigma \in\left[L^{2}(\Omega)\right]^{9}, D \in\left[L^{2}(\Omega)\right]^{3}$ and subsequences $\left\{\sigma_{i j}(h)(u(h)\right.$, $\varphi(h))\}_{h>0}$ and $\left\{D_{i}(h)(u(h), \varphi(h))\right\}_{h>0}$ (still indexed by $h$ ), such that the following weak convergences are satisfied, when $h \rightarrow 0^{+}$,

$$
\begin{gathered}
\sigma_{i j}(h)(u(h), \varphi(h)) \rightarrow \sigma_{i j}=C_{i j l m} \kappa_{l m}+P_{\alpha i j} \partial_{\alpha} \varphi \text { in }\left[L^{2}(\Omega)\right]^{9}, \\
D_{i}(h)(u(h), \varphi(h)) \rightarrow D_{i}=P_{i l m} \kappa_{l m}-\varepsilon_{i \alpha} \partial_{\alpha} \varphi \text { in }\left[L^{2}(\Omega)\right]^{3},
\end{gathered}
$$

with

$$
\begin{aligned}
\sigma_{\alpha \beta} & =(-1)^{\alpha^{*}} \partial_{\alpha^{*}} \theta_{\beta}, \\
\sigma_{\alpha 3} & =(-1)^{\alpha^{*}} \partial_{\alpha^{*}} \theta_{3}, \\
\sigma_{33} & =c e_{33}(u)+p_{\alpha 3} \partial_{\alpha} \varphi+r\left(\theta_{1}, \theta_{2}\right),
\end{aligned}
$$

where $\alpha^{*}=2$, if $\alpha=1$ and $\alpha^{*}=1$, if $\alpha=2$, and

$$
D_{i}=p_{i 3} e_{33}(u)-(p \varepsilon)_{i \beta} \partial_{\beta} \varphi+s_{i}(\theta) .
$$


The coefficients, $c, p_{i 3}$ and $(p \varepsilon)_{i \alpha}$, are related, respectively, to the elasticity matrix $\mathcal{C}$, to the piezoelectric coefficients $P_{i j k}$, and to both the piezoelectric and dielectric coefficients $P_{i j k}$ and $\varepsilon_{i j}$ by the following formulas:

$$
\begin{aligned}
& c=\frac{\operatorname{det} \mathcal{C}}{\operatorname{det} \mathcal{M} \operatorname{det} \mathcal{N}}, \quad c=c\left(x_{1}, x_{2}, x_{3}\right), \\
& p_{i 3}=P_{i 33}-\frac{1}{\operatorname{det} \mathcal{M}} \operatorname{det}\left[\begin{array}{cccc}
C_{3311} & C_{3312} & C_{3322} & 0 \\
& & P_{i 11} \\
& \mathcal{M} & P_{i 12} \\
& & P_{i 22}
\end{array}\right] \text {, }
\end{aligned}
$$

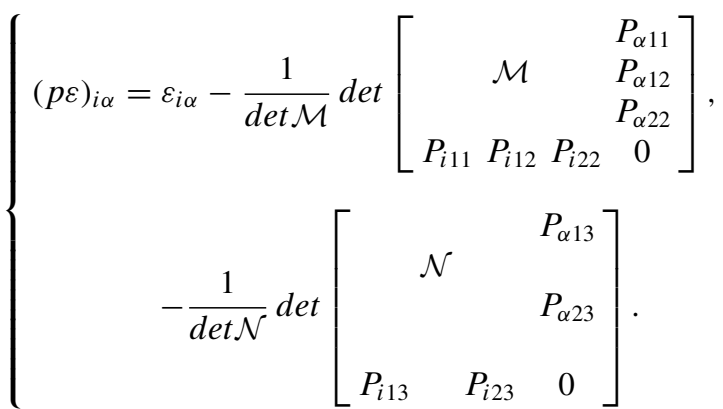

The coefficients $r\left(\theta_{1}, \theta_{2}\right)$ and $s_{i}(\theta)$ are defined by

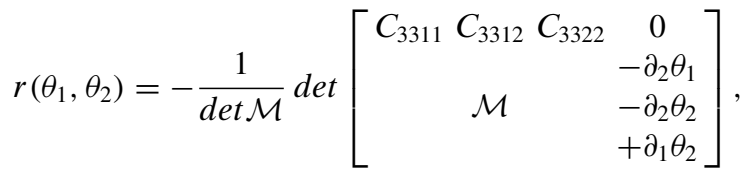

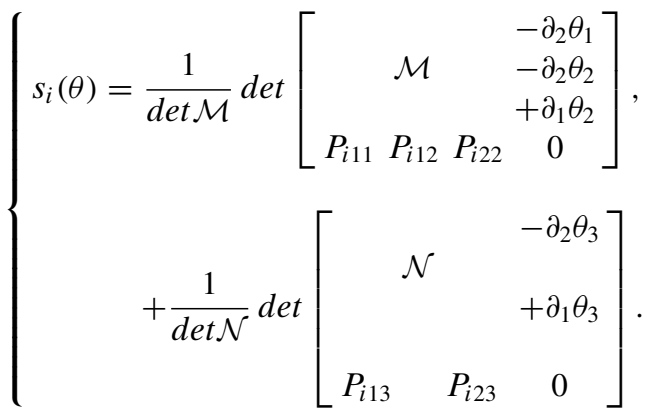

Proof. From (31) and the weak convergence (40) we deduce easily the formulas indicated in (55) for $\sigma_{i j}$ and $D_{i}$. From (50) and (53) it follows (56). Introducing in (55) the definition of $\kappa$ given by (41)-(46), we obtain the expressions indicated for $\sigma_{33}$ and $D_{i}$.

The next result describes the structure of the variational formulation of the limit rod model. 
THEOREM 3.3. The triple $(u, \varphi, \theta)$, where $(u, \varphi)$ is a weak limit of the sequence $(u(h), \varphi(h))$ and $\theta=\left(\theta_{1}, \theta_{2}, \theta_{3}\right)$ is the function of $u, \varphi$ and $\kappa$ found in Theorem 3.1, is the solution of the following variational problem:

$$
\left\{\begin{array}{l}
\text { Find }(u, \varphi) \in V_{B N} \times \Psi_{l} \text { and } \theta\left(., ., x_{3}\right) \in H^{1}(\omega), \quad \text { such that: } \\
a((u, \varphi, \theta),(v, \psi))=l(v, \psi), \quad \forall(v, \psi) \in V_{B N} \times \Psi_{l 0}, \\
\varphi=\varphi_{0}, \quad \text { on } \Gamma_{e D},
\end{array}\right.
$$

where

$$
\begin{aligned}
& {\left[\begin{array}{l}
a((u, \varphi, \theta),(v, \psi)) \equiv \int_{\Omega} c e_{33}(u) e_{33}(v) d x+\int_{\Omega}(p \varepsilon)_{\alpha \beta} \partial_{\alpha} \varphi \partial_{\beta} \psi d x \\
+\int_{\Omega} p_{\alpha 3}\left[e_{33}(v) \partial_{\alpha} \varphi-e_{33}(u) \partial_{\alpha} \psi\right] d x+\int_{\Omega} r\left(\theta_{1}, \theta_{2}\right) e_{33}(v) d x-\int_{\Omega} s_{\alpha}(\theta) \partial_{\alpha} \psi d x \\
=\int_{\Omega} \sigma_{33} e_{33}(v) d x-\int_{\Omega} D_{\alpha} \partial_{\alpha} \psi d x,
\end{array}\right.} \\
& {\left[\begin{array}{l}
C_{i j \alpha \beta} \kappa_{i j}+P_{\rho \alpha \beta} \partial_{\rho} \varphi=(-1)^{\alpha^{*}} \partial_{\alpha^{*}} \theta_{\beta}, \\
C_{i j 3 \alpha} \kappa_{i j}+P_{\rho 3 \alpha} \partial_{\rho} \varphi=(-1)^{\alpha^{*}} \partial_{\alpha^{*}} \theta_{3},
\end{array}\right.}
\end{aligned}
$$

with $\alpha^{*}=2$ if $\alpha=1$ and $\alpha^{*}=1$ if $\alpha=2$, and $\kappa_{i j}$ are the components of the weak limit $\kappa$ defined in (41)-(46), and

$$
l(v, \psi) \equiv \int_{\Omega} f \cdot v d x+\int_{\Gamma_{N}} g \cdot v d \Gamma_{N} .
$$

Proof. Considering $(v, \psi) \in V_{B N} \times \Psi$ in (32), so in particular $e_{\alpha \beta}(v)=e_{3 \beta}(v)=0$, and taking the limit when $h \rightarrow 0^{+}$we directly obtain

$$
\left\{\begin{array}{l}
\int_{\Omega} C_{i j 33} \kappa_{i j} e_{33}(v) d x+\int_{\Omega} \varepsilon_{\alpha \beta} \partial_{\alpha} \varphi \partial_{\beta} \psi d x+ \\
\int_{\Omega} P_{\alpha 33} \partial_{\alpha} \varphi e_{33}(v) d x-\int_{\Omega} P_{\alpha i j} \partial_{\alpha} \psi \kappa_{i j} d x=\int_{\Omega} f \cdot v d x+\int_{\Gamma_{N}} g \cdot v d \Gamma_{N} .
\end{array}\right.
$$

Using the definitions of $\sigma_{33}$ and $D_{\alpha}$ given in (55), we get the variational formulation (60) for all $(v, \psi) \in V_{B N} \times \Psi$. But, as $\mathcal{D}(\Omega)$ is dense in $\Psi_{l 0}$ (cf. Proposition 3.3), and $\mathcal{D}(\Omega) \subset \Psi$, we conclude that $\Psi$ is also dense in $\Psi_{l 0}$. So, by a density argument we get (60) for all $(v, \psi) \in V_{B N} \times \Psi_{l 0}$.

\section{The Generalized Piezoelectric Bernoulli-Navier Rod Model}

This section describes the differential formulation of the limit rod model (60). With this objective it is necessary to first introduce additional notation and new functions. 
The limit rod mechanical displacement $u$ belongs to the space $V_{B N}$ defined in (36); therefore it satisfies

$$
\begin{aligned}
u(x) & =\left(u_{1}(x), u_{2}(x), u_{3}(x)\right), \quad x=\left(x_{1}, x_{2}, x_{3}\right) \in \Omega, \\
u_{\alpha}(x) & =\xi_{\alpha}\left(x_{3}\right), \quad \xi_{\alpha} \in H_{0}^{2}(] 0, L[), \quad \alpha=1,2, \\
u_{3}(x) & =\xi_{3}\left(x_{3}\right)-x_{\alpha} \partial_{3} \xi_{\alpha}\left(x_{3}\right), \quad \xi_{3} \in H_{0}^{1}(] 0, L[), \\
e_{33}(u)(x) & =\partial_{3} \xi_{3}\left(x_{3}\right)-x_{\alpha} \partial_{33} \xi_{\alpha}\left(x_{3}\right),
\end{aligned}
$$

where $\xi_{\alpha}$ and $\xi_{3}$ are, respectively, the bending and the stretching components of $u$. We also introduce the axial stress component

$$
\begin{aligned}
q & =q(u, \varphi)=\int_{\omega} \sigma_{33} d \omega=\int_{\omega}\left[c e_{33}(u)+p_{\alpha 3} \partial_{\alpha} \varphi+r\left(\theta_{1}, \theta_{2}\right)\right] d \omega \\
& =\int_{\omega}\left[c\left(\partial_{3} \xi_{3}-x_{\alpha} \partial_{33} \xi_{\alpha}\right)+p_{\alpha 3} \partial_{\alpha} \varphi+r\left(\theta_{1}, \theta_{2}\right)\right] d \omega
\end{aligned}
$$

the bending moment components

$$
\begin{aligned}
m_{\beta} & =m_{\beta}(u, \varphi)=\int_{\omega} x_{\beta} \sigma_{33} d \omega \\
& =\int_{\omega}\left[c\left(x_{\beta} \partial_{3} \xi_{3}-x_{\beta} x_{\alpha} \partial_{33} \xi_{\alpha}\right)+x_{\beta} p_{\alpha 3} \partial_{\alpha} \varphi+x_{\beta} r\left(\theta_{1}, \theta_{2}\right)\right] d \omega
\end{aligned}
$$

the functions $F_{i}$ and $M_{\beta}$, associated to the applied mechanical loadings,

$$
\begin{aligned}
F_{i} & =F_{i}\left(x_{3}\right)=\int_{\omega} f_{i}\left(x_{1}, x_{2}, x_{3}\right) d \omega+\int_{\gamma} g_{i}\left(x_{1}, x_{2}, x_{3}\right) d \gamma \\
M_{\beta} & =M_{\beta}\left(x_{3}\right)=\int_{\omega} x_{\beta} f_{3}\left(x_{1}, x_{2}, x_{3}\right) d \omega+\int_{\gamma} x_{\beta} g_{3}\left(x_{1}, x_{2}, x_{3}\right) d \gamma
\end{aligned}
$$

and finally the functions $I_{0}, I_{\alpha}$ and $H_{\alpha \beta}$,

$$
\begin{aligned}
I_{0} & =I_{0}\left(x_{3}\right)=\int_{\omega} c d \omega, \quad I_{\alpha}=I_{\alpha}\left(x_{3}\right)=\int_{\omega} x_{\alpha} c d \omega, \\
H_{\alpha \beta} & =H_{\alpha \beta}\left(x_{3}\right)=\int_{\omega} x_{\alpha} x_{\beta} c d \omega .
\end{aligned}
$$

\subsection{The Limit Model Defined in the Scaled Rod}

The next theorem formulates the variational limit problem (60) as a system of two coupled boundary value problems formulated in the scaled $\operatorname{rod} \Omega=\omega \times(0, L)$. 
THEOREM 4.1. The solution $(u, \varphi)$ of $(60)$ satisfies

$$
\begin{aligned}
& -\partial_{3}\left(I_{0} \partial_{3} \xi_{3}-I_{\alpha} \partial_{33} \xi_{\alpha}\right)=F_{3}+\partial_{3}\left(\int_{\omega}\left(p_{\alpha 3} \partial_{\alpha} \varphi+r\left(\theta_{1}, \theta_{2}\right)\right) d \omega\right) \quad \text { in }(0, L), \\
& \left\{\begin{array}{l}
\partial_{33}\left(-I_{\beta} \partial_{3} \xi_{3}+H_{\alpha \beta} \partial_{33} \xi_{\alpha}\right)= \\
F_{\beta}+\partial_{3} M_{\beta}+\partial_{33}\left(\int_{\omega}\left(x_{\beta} p_{\alpha 3} \partial_{\alpha} \varphi+x_{\beta} r\left(\theta_{1}, \theta_{2}\right)\right) d \omega\right) \quad \text { in }(0, L), \quad \text { for } \beta=1,2,
\end{array}\right.
\end{aligned}
$$

$$
\xi_{3}(0)=\xi_{3}(L)=0
$$

$$
\begin{gathered}
\xi_{\alpha}(0)=\partial_{3} \xi_{\alpha}(0)=0, \quad \xi_{\alpha}(L)=\partial_{3} \xi_{\alpha}(L)=0, \\
\partial_{\alpha}(\underbrace{-(p \varepsilon)_{\alpha \beta} \partial_{\beta} \varphi+p_{\alpha 3} e_{33}(u)+s_{\alpha}(\theta)}_{D_{\alpha}})=0 \quad \text { in } \quad \Omega, \\
D_{\alpha} v_{\alpha}=0 \quad \text { on } \quad \Gamma_{e N}, \\
\varphi=\varphi_{0} \quad \text { on } \quad \Gamma_{e D},
\end{gathered}
$$

$$
\left[\begin{array}{l}
C_{i j \alpha \beta} \kappa_{i j}+P_{\rho \alpha \beta} \partial_{\rho} \varphi=(-1)^{\alpha^{*}} \partial_{\alpha^{*}} \theta_{\beta}, \\
C_{i j 3 \alpha} \kappa_{i j}+P_{\rho 3 \alpha} \partial_{\rho} \varphi=(-1)^{\alpha^{*}} \partial_{\alpha^{*}} \theta_{3},
\end{array}\right.
$$

with $\alpha^{*}=2$ if $\alpha=1$ and $\alpha^{*}=1$ if $\alpha=2$, and $\kappa_{i j}$ are the components of the weak limit $\kappa$ found in Theorem 3.1.

Proof. We first notice that (71)-(72) and (74)-(75) are the boundary conditions for $u$ and $\varphi$, respectively.

Considering $v=0$ in (60) we obtain

$$
\int_{\Omega}(\underbrace{-(p \varepsilon)_{\alpha \beta} \partial_{\beta} \varphi+p_{\alpha 3} e_{33}(u)+s_{\alpha}(\theta)}_{D_{\alpha}}) \partial_{\alpha} \psi d x=0,
$$

and, applying Green's formula, this equation leads to

$$
-\int_{\Omega} \partial_{\alpha} D_{\alpha} \psi d x+\int_{\Gamma_{e N}} D_{\alpha} v_{\alpha} \psi d \Gamma_{e N}=0,
$$

which immediately gives formulas (73)-(74).

Choosing now $\psi=0$ in (60), we get

$$
\int_{\Omega}(\underbrace{c e_{33}(u)+p_{\alpha 3} \partial_{\alpha} \varphi+r\left(\theta_{1}, \theta_{2}\right)}_{\sigma_{33}(u, \varphi)}) e_{33}(v) d x=\int_{\Omega} f \cdot v d x+\int_{\Gamma_{N}} g \cdot v d \Gamma_{N} .
$$


Considering in (79) $v=\left(0,0, \eta_{3}\right) \in V_{B N}\left(\eta_{3} \in H_{0}^{1}(] 0, L[)\right)$, the formula (79) becomes

$$
\begin{aligned}
\int_{0}^{L} & {\left[(\underbrace{\int_{\omega} c d \omega}_{I_{0}}) \partial_{3} \xi_{3}-(\underbrace{\int_{\omega} c x_{\alpha} d \omega}_{I_{\alpha}}) \partial_{33} \xi_{\alpha}+\int_{\omega}\left(p_{\alpha 3} \partial_{\alpha} \varphi+r\left(\theta_{1}, \theta_{2}\right)\right) d \omega\right] \partial_{3} \eta_{3} d x_{3} } \\
& =\int_{0}^{L} F_{3} \eta_{3} d x_{3},
\end{aligned}
$$

which clearly gives (69), after the application of Green's formula with respect to the variable $x_{3}$. Then, choosing $v \in V_{B N}$ in (79), with the stretching component $\eta_{3}=0$, and such that $v=\left(\eta_{1}, 0,-x_{1} \partial_{3} \eta_{1}\right)$ or $v=\left(0, \eta_{2},-x_{2} \partial_{3} \eta_{2}\right)\left(\eta_{\alpha} \in H_{0}^{2}(] 0, L[)\right)$, the formula (79) changes to

$$
\begin{aligned}
\int_{0}^{L} & {\left[(\underbrace{\int_{\omega} c x_{\beta} d \omega}_{I_{\beta}}) \partial_{3} \xi_{3}-(\underbrace{\int_{\omega} c x_{\alpha} x_{\beta} d \omega}_{H_{\alpha \beta}}) \partial_{33} \xi_{\alpha}+\int_{\omega}\left(x_{\beta} p_{\alpha 3} \partial_{\alpha} \varphi+x_{\beta} r\left(\theta_{1}, \theta_{2}\right)\right) d \omega\right] } \\
& \times\left(-\partial_{33} \eta_{\beta}\right) d x_{3} \\
= & \left.\int_{0}^{L}\left(-M_{\beta} \partial_{3} \eta_{\beta}+F_{\beta} \eta_{\beta}\right) d x_{3} \quad \text { (with no sum on } \beta\right),
\end{aligned}
$$

which gives (70), for $\beta=1,2$, after the application of Green's formula with respect to the variable $x_{3}$.

Using the definitions of the axial stress component $q$ and the bending moments components $m_{\beta}$, the system (69)-(76) can be rewritten in the following form

$$
\begin{gathered}
-\partial_{3} q=F_{3} \quad \text { in }(0, L), \\
-\partial_{33} m_{\beta}=F_{\beta}+\partial_{3} M_{\beta} \quad \text { in } \quad(0, L), \quad \text { for } \beta=1,2, \\
\xi_{3}(0)=\xi_{3}(L)=0, \\
\xi_{\alpha}(0)=\partial_{3} \xi_{\alpha}(0)=0, \quad \xi_{\alpha}(L)=\partial_{3} \xi_{\alpha}(L)=0, \\
\partial_{\alpha} D_{\alpha}=0 \quad \text { in } \quad \Omega, \\
D_{\alpha} v_{\alpha}=0 \quad \text { on } \quad \Gamma_{e N}, \\
\varphi=\varphi_{0} \quad \text { on } \quad \Gamma_{e D} . \\
{\left[\begin{array}{c}
C_{i j \alpha \beta} \kappa_{i j}+P_{\rho \alpha \beta} \partial_{\rho} \varphi \\
C_{i j 3 \alpha} \kappa_{i j}+P_{\rho 3 \alpha} \partial_{\rho} \varphi
\end{array}=(-1)^{\alpha^{*}} \partial_{\alpha^{*}} \theta_{\beta}, \quad \text { in } \quad \Omega,\right.}
\end{gathered}
$$

with $\alpha^{*}=2$ if $\alpha=1$ and $\alpha^{*}=1$ if $\alpha=2$, and $\kappa_{i j}$ are the components of the weak limit $\kappa$ found in Theorem 3.1.

Moreover it is interesting to remark that the system (69)-(72) constitutes a generalization of the Bernoulli-Navier equations for the anisotropic nonhomogeneous case (cf. Trabucho and Viaño [13], page 678 formula (23.60)) and (73)-(75) 
correspond to reduced Maxwell-Gauss equations. Moreover in the following corollary, we particularize (69)-(76) for a homogeneous isotropic material with constant piezoelectric and dielectric coefficients.

COROLLARY 4.1. If the piezoelectric and dielectric coefficients are constants, and for homogeneous and isotropic elastic coefficients (cf. (16)), the coefficients $\kappa_{\alpha \beta}$ in (41)-(46) and $c, p_{\alpha 3},(p \varepsilon)_{\alpha \beta}$ defined in (58) change to

$$
\begin{aligned}
& \left\{\begin{array}{l}
\kappa_{\alpha \beta}=\left[-\frac{1}{2 \mu} P_{\rho \alpha \beta}+\frac{\lambda}{4 \mu(\lambda+\mu)} P_{\rho \tau \tau} \delta_{\alpha \beta}\right] \partial_{\rho} \varphi-\frac{\lambda}{2(\lambda+\mu)} e_{33}(u) \delta_{\alpha \beta}+ \\
\frac{1}{2 \mu}(-1)^{\alpha^{*}} \partial_{\alpha^{*}} \theta_{\beta}-\frac{\lambda}{4 \mu(\lambda+\mu)\left(\sum_{\rho=1}^{2}(-1)^{\rho^{*}} \partial_{\rho^{*} *} \theta_{\rho}\right) \delta_{\alpha \beta}},
\end{array}\right. \\
& \kappa_{3 \beta}=-\frac{1}{2 \mu} P_{\rho 3 \beta} \partial_{\rho} \varphi+\frac{1}{2 \mu}(-1)^{\beta^{*}} \partial_{\beta^{*}} \theta_{3}, \\
& \kappa_{33}=e_{33}(u),
\end{aligned}
$$

with $\alpha^{*}, \beta^{*}, \rho^{*}=2$ if $\alpha, \beta, \rho=1$ and $\alpha^{*}, \beta^{*}, \rho^{*}=1$ if $\alpha, \beta, \rho=2$, and

$$
\begin{aligned}
c & =\frac{\mu(3 \lambda+2 \mu)}{\lambda+\mu} \quad(\text { Young's modulus of the material), } \\
p_{\alpha 3} & =P_{\alpha 33}-\frac{\lambda}{2(\lambda+\mu)} P_{\alpha \rho \rho}, \\
(p \varepsilon)_{\alpha \beta} & =\varepsilon_{\alpha \beta}+\frac{1}{2 \mu} P_{\alpha \rho \tau} P_{\beta \rho \tau}-\frac{\lambda}{4 \mu(\lambda+\mu)} P_{\alpha \rho \rho} P_{\beta \tau \tau}+\frac{1}{\mu} P_{\alpha 3 \rho} P_{\beta 3 \rho} .
\end{aligned}
$$

Moreover, $r\left(\theta_{1}, \theta_{2}\right)$ and $s_{\alpha}(\theta)$ defined in (59) become

$$
\begin{aligned}
& r\left(\theta_{1}, \theta_{2}\right)=\frac{\lambda}{2(\lambda+\mu)} \partial_{2} \theta_{1}-\frac{\lambda}{2(\lambda+\mu)} \partial_{1} \theta_{2}, \\
& \left\{\begin{array}{l}
s_{\alpha}(\theta)=\left(\frac{-\lambda}{4 \mu(\lambda+\mu) P_{\alpha 22}}+\frac{\lambda+2 \mu}{4 \mu(\lambda+\mu)} P_{\alpha 11}\right) \partial_{2} \theta_{1}+\frac{1}{\mu} P_{\alpha 12} \partial_{2} \theta_{2} \\
-\left(\frac{\lambda+2 \mu}{4 \mu(\lambda+\mu)} P_{\alpha 22}-\frac{\lambda}{4 \mu(\lambda+\mu)} P_{\alpha 11}\right) \partial_{1} \theta_{2}+\frac{1}{\mu} P_{\alpha 13} \partial_{2} \theta_{3}-\frac{1}{\mu} P_{\alpha 23} \partial_{1} \theta_{3},
\end{array}\right.
\end{aligned}
$$

and finally

$$
\begin{aligned}
I_{0} & =\frac{\mu(3 \lambda+2 \mu)}{\lambda+\mu} \operatorname{meas}(\omega), \quad I_{\beta}=0, \quad H_{\alpha \beta}=0, \quad \text { for } \alpha \neq \beta, \\
H_{\alpha \alpha} & =I_{0} \int_{\omega} x_{\alpha}^{2} d \omega \quad(\text { no sum on } \alpha) .
\end{aligned}
$$


Consequently, for this particular case, the system (69)-(72) becomes

$$
\begin{aligned}
& -\partial_{3}\left(I_{0} \partial_{3} \xi_{3}\right)=F_{3}+\partial_{3}\left(\int_{\omega}\left(p_{\alpha 3} \partial_{\alpha} \varphi+r\left(\theta_{1}, \theta_{2}\right)\right) d \omega\right) \text { in }(0, L), \\
& \left\{\begin{array}{l}
\partial_{33}\left(H_{\beta \beta} \partial_{33} \xi_{3}\right)=F_{\beta}+\partial_{3} M_{\beta}+ \\
\partial_{33}\left(\int_{\omega}\left(x_{\beta} p_{\alpha 3} \partial_{\alpha} \varphi+x_{\beta} r\left(\theta_{1}, \theta_{2}\right)\right) d \omega\right) \text { in }(0, L), \text { for } \beta=1,2,
\end{array}\right. \\
& \xi_{3}(0)=\xi_{3}(L)=0 \\
& \xi_{\alpha}(0)=\partial_{3} \xi_{\alpha}(0)=0, \quad \xi_{\alpha}(L)=\partial_{3} \xi_{\alpha}(L)=0, \\
& -(p \varepsilon)_{\alpha \beta} \partial_{\alpha \beta} \varphi-p_{\alpha 3} \partial_{33} \xi_{\alpha}+\partial_{\alpha} s_{\alpha}(\theta)=0 \quad \text { in } \quad \Omega \\
& (\underbrace{-(p \varepsilon)_{\alpha \beta} \partial_{\beta} \varphi+p_{\alpha 3}\left(\partial_{3} \xi_{3}-x_{\rho} \partial_{33}\left(\xi_{\rho}\right)+s_{\alpha}(\theta)\right.}_{D_{\alpha}}) v_{\alpha}=0 \quad \text { on } \quad \Gamma_{e N}, \\
& \varphi=\varphi_{0} \quad \text { on } \quad \Gamma_{e D} \\
& {\left[\begin{array}{l}
C_{i j \alpha \beta} \kappa_{i j}+P_{\rho \alpha \beta} \partial_{\rho} \varphi=(-1)^{\alpha^{*}} \partial_{\alpha^{*}} \theta_{\beta}, \\
C_{i j 3 \alpha} \kappa_{i j}+P_{\rho 3 \alpha} \partial_{\rho} \varphi=(-1)^{\alpha^{*}} \partial_{\alpha^{*}} \theta_{3},
\end{array}\right.}
\end{aligned}
$$

with $\alpha^{*}=2$ if $\alpha=1$ and $\alpha^{*}=1$ if $\alpha=2$, and $\kappa_{i j}$ are the components of the weak limit $\kappa$ found in Theorem 3.1.

Proof. It is enough to introduce the definition of $C_{i j k l}$, cf. (15)-(16), in formulas (41)-(46) and (58) and to use the fact that $P_{i j k l}$ and $\varepsilon_{i j}$ are constants, thus independent of $x=\left(x_{1}, x_{2}, x_{3}\right)$.

We remark that the system (69)-(76) (or (94)-(101)) is a coupled problem, where the two unknowns $u$ and $\varphi$ are interdependent. We were not able to determine the exact expression of the limit rod electric potential, as a function of the limit rod displacement vector, unlike the plate case (cf. Figueiredo and Leal [7], or Sene [12]), since the equation (73) (or (98)) is more complex than in the plate case. This equation involves the third unknown $\theta$ and the derivatives with respect to $x_{1}$ and $x_{2}$. However, if the elastic, piezoelectric and dielectric coefficients satisfy the hypotheses of Corollary 4.1, then formula (98) shows that the limit electric potential $\varphi$ depends explicitly on the bending components $\xi_{\alpha}$ of the limit displacement $u$ and on $\theta$, and is independent of the stretching component.

\subsection{The Limit Model Defined in the Original Rod}

To interpret the limit problem, with respect to the original rod $\Omega^{h}=\omega^{h} \times(0, L)$, for a fixed $h$ small enough, it is convenient to formulate (69)-(75) in $\Omega^{h}$. To achieve this, we define $\xi_{1}^{h}, \xi_{2}^{h}, \xi_{3}^{h}, z^{h}=\left(z_{1}^{h}, z_{2}^{h}, z_{3}^{h}\right), \phi^{h}, \theta^{h}$ and $\kappa^{h}$ related, respectively, to the limit 
mechanical displacement $u$, to the limit electric potential $\varphi$, to the function $\theta$, and to $\kappa$ by the re-scalings

$$
\begin{aligned}
& \xi^{h}=\left(\xi_{1}^{h}, \xi_{2}^{h}, \xi_{3}^{h}\right), \quad \xi_{\alpha}^{h}=h^{-1} \xi_{\alpha} \quad \text { and } \quad \xi_{3}^{h}=\xi_{3}, \quad \text { in } \quad[0, L], \\
& z_{\alpha}^{h}\left(x^{h}\right)=h^{-1} u_{\alpha}(x) \text { and } z_{3}^{h}\left(x^{h}\right)=u_{3}(x) \text {, } \\
& \phi^{h}\left(x^{h}\right)=h \varphi(x) \quad \text { and } \quad \theta^{h}\left(x^{h}\right)=\left(h \theta_{1}^{h}\left(x^{h}\right), h \theta_{2}^{h}\left(x^{h}\right), h \theta_{3}^{h}\left(x^{h}\right)\right)=h \theta(x) \text {, } \\
& \kappa_{\alpha \beta}^{h}\left(x^{h}\right)=\kappa_{\alpha \beta}(x), \quad \kappa_{\alpha 3}^{h}\left(x^{h}\right)=\kappa_{\alpha 3}(x), \quad \text { and } \quad \kappa_{33}^{h}\left(x^{h}\right)=\kappa_{33}(x) \text {, }
\end{aligned}
$$

for all $x^{h}=\left(x_{1}^{h}, x_{2}^{h}, x_{3}\right)$, with $x_{\alpha}^{h}=h x_{\alpha}$ and $x=\left(x_{1}, x_{2}, x_{3}\right) \in \Omega=\omega \times(0, L)$. The components $\xi_{\alpha}^{h}$ and $\xi_{3}^{h}$ are respectively the bending and stretching components of the mechanical displacement of the $\operatorname{rod} \Omega^{h}=\omega^{h} \times(0, L)$. The functions $z_{i}^{h}$ and $\phi^{h}$ are, respectively, the limit mechanical displacement and limit electric potential of the $\operatorname{rod} \Omega^{h}$.

We can now state the following immediate consequence of Theorem 4.1.

THEOREM 4.2. The re-scaled limit displacement $z^{h}\left(x^{h}\right)=\left(\xi_{1}^{h}\left(x_{3}\right), \xi_{2}^{h}\left(x_{3}\right), \xi_{3}^{h}\left(x_{3}\right)-\right.$ $\left.x_{\alpha}^{h} \partial_{3} \xi_{\alpha}^{h}\left(x_{3}\right)\right)$ and the re-scaled limit electric potential $\phi^{h}\left(x^{h}\right)$ are the solution of the system

$$
\begin{gathered}
-\partial_{3}\left(I_{0}^{h} \partial_{3} \xi_{3}^{h}-I_{\alpha}^{h} \partial_{33} \xi_{\alpha}^{h}\right)=F_{3}^{h}+\partial_{3}\left(\int_{\omega^{h}}\left(p_{\alpha 3} \partial_{\alpha} \phi^{h}+r\left(\theta_{1}^{h}, \theta_{2}^{h}\right)\right) d \omega^{h}\right) \text { in }(0, L), \\
\left\{\begin{array}{l}
\partial_{33}\left(-I_{\beta}^{h} \partial_{3} \xi_{3}^{h}+H_{\alpha \beta}^{h} \partial_{33} \xi_{\alpha}^{h}\right) \\
=F_{\beta}^{h}+\partial_{3} M_{\beta}^{h}+\partial_{33}\left(\int_{\omega^{h}}\left(x_{\beta}^{h} p_{\alpha 3} \partial_{\alpha} \phi^{h}+x_{\beta}^{h} r\left(\theta_{1}^{h}, \theta_{2}^{h}\right)\right) d \omega^{h}\right) \quad \text { in }(0, L), \text { for } \beta=1,2,
\end{array}\right.
\end{gathered}
$$

$$
\begin{gathered}
\xi_{3}^{h}(0)=\xi_{3}^{h}(L)=0, \\
\xi_{\alpha}^{h}(0)=\partial_{3} \xi_{\alpha}^{h}(0)=0, \quad \xi_{\alpha}^{h}(L)=\partial_{3} \xi_{\alpha}^{h}(L)=0, \\
\partial_{\alpha}^{h}(\underbrace{-(p \varepsilon)_{\alpha \beta} \partial_{\beta}^{h} \phi^{h}+p_{\alpha 3} e_{33}\left(z^{h}\right)+s_{\alpha}\left(\theta^{h}\right)}_{D_{\alpha}})=0 \quad \text { in } \quad \Omega^{h}, \\
D_{\alpha} v_{\alpha}^{h}=0 \\
\phi^{h}=\varphi_{0}^{h} \quad \text { on } \Gamma_{e N}^{h}, \\
\Gamma_{e D}^{h}, \\
{\left[\begin{array}{c}
C_{i j \alpha \beta} \kappa_{i j}^{h}+P_{\rho \alpha \beta} \partial_{\rho}^{h} \phi^{h}=(-1)^{\alpha^{*}} \partial_{\alpha^{*}}^{h} \theta_{\beta}^{h}, \\
C_{i j 3 \alpha} \kappa_{i j}^{h}+P_{\rho 3 \alpha} \partial_{\rho}^{h} \phi^{h}=(-1)^{\alpha^{*}} \partial_{\alpha^{*}}^{h} \theta_{3}^{h},
\end{array}\right.}
\end{gathered}
$$


$\left(\alpha^{*}=2\right.$, if $\alpha=1$ and $\alpha^{*}=1$, if $\left.\alpha=2\right)$ where the functions $F_{i}^{h}$ and $M_{\beta}^{h}$, which are associated with the original applied mechanical loadings, are defined by

$$
\begin{aligned}
& F_{i}^{h}=F_{i}^{h}\left(x_{3}\right)=\int_{\omega^{h}} f_{i}^{h}\left(x_{1}^{h}, x_{2}^{h}, x_{3}\right) d \omega^{h}+\int_{\gamma^{h}} g_{i}^{h}\left(x_{1}^{h}, x_{2}^{h}, x_{3}\right) d \gamma^{h}, \\
& M_{\beta}^{h}=M_{\beta}^{h}\left(x_{3}\right)=\int_{\omega^{h}} x_{\beta}^{h} f_{3}^{h}\left(x_{1}^{h}, x_{2}^{h}, x_{3}\right) d \omega+\int_{\gamma^{h}} x_{\beta}^{h} g_{3}^{h}\left(x_{1}^{h}, x_{2}^{h}, x_{3}\right) d \gamma^{h},
\end{aligned}
$$

and the functions $I_{0}^{h}, I_{\alpha}^{h}$ and $H_{\alpha \beta}^{h}$ are defined by

$$
\begin{aligned}
I_{0}^{h} & =I_{0}^{h}\left(x_{3}\right)=\int_{\omega^{h}} c d \omega^{h}, \quad I_{\alpha}^{h}=I_{\alpha}^{h}\left(x_{3}\right)=\int_{\omega^{h}} x_{\alpha}^{h} c d \omega^{h}, \\
H_{\alpha \beta}^{h} & =H_{\alpha \beta}^{h}\left(x_{3}\right)=\int_{\omega^{h}} x_{\alpha}^{h} x_{\beta}^{h} c d \omega^{h} .
\end{aligned}
$$

Acknowledgments This work is partially supported by the project Mathematical analysis of piezoelectric problems (FCT-POCI/MAT/59502/2004 of Portugal), and is part of the project New materials, adaptive systems and their nonlinearities; modelling, control and numerical simulation (European Community program HRN-CT-2002-00284). The authors are grateful to Professor Juan Viaño for point out the use of theorem "div-curl" to obtain the Equations (50) and (53).

\section{References}

1. Bernadou, M., Haenel, C.: Modelization and numerical approximation of piezoelectric thin shells. I. The continuous problems. Comput. Methods Appl. Mech. Eng. 192(37-38), 4003-4030 (2003)

2. Ciarlet, P.: Mathematical Elasticity, Volume II: Theory of Plates. North-Holland, Amsterdam (1997)

3. Collard, C., Miara, B.: Two-dimensional models for geometrically nonlinear thin piezoelectric shells. Asymptot. Anal. 31(2), 113-151 (2003)

4. Eringen, A.C., Maugin, G.: Electrodynamics of Continua, Volume I: Foundations and Solid Media. Springer, Berlin Heidelberg New York (1990)

5. Green, A.E., Zerna, W.: Theoretical Elasticity, 2nd edn. Oxford, New York (1968)

6. Girault, V., Raviart, P.A.: Finite Element Approximation of the Navier-Stokes Equations, Lectures Notes in Mathematics 749. Springer, Berlin Heidelberg New York (1979)

7. Figueiredo, I.N., Leal, C.F.: A piezoelectric anisotropic plate model. Asymptot. Anal. 44(3-4), 327-346 (2005)

8. Ikeda, T.: Fundamentals of Piezoelectricity. Oxford University Press, London, UK (1990)

9. Maugin, G.A., Attou, D.: An asymptotic theory of thin piezoelectric plates. Q. J. Mech. Appl. Math. 43, 347-362 (1990)

10. Rahmoune, M., Benjeddou, A., Ohayon, R.: New thin piezoelectric plate models. J. Intell. Mater. Syst. Struct. 9, 1017-1029 (1998)

11. Raoult, A., Sene, A.: Modelling of piezoelectric plates including magnetic effects. Asymptot. Anal. 34(1), 1-40 (2003)

12. Sene, A.: Modelling of piezoelectric static thin plates. Asymptot. Anal. 25(1), 1-20 (2001)

13. Trabucho, L., Viaño, J.M.: Mathematical modelling of rods. In: Ciarlet, P.G., Lions, J.L. (eds.) Handbook of Numerical Analysis, vol. 4, pp. 487-974. North-Holland, Amsterdam (1996)

14. Young, E.C.: Vector and Tensor Analysis, Monographs and Textbooks in Pure and Applied Mathematics 48. Marcel Dekker, New York (1978) 